\title{
HEALING OF CHRONIC APICAL PERIODONTITIS USING NOVEL BIOCERAMIC SEALER BIOROOT RCS
}

\author{
Kostadin Zhekov, Vesela Stefanova \\ Department of Operative Dentistry and Endodontics, Faculty of Dental Medicine, \\ Medical University - Plovdiv, Bulgaria.
}

\section{ABSTRACT:}

Introduction: Periapical radiolucency is a sign of an inflammatory process in the bone, surrounding the apex of the root. The healing of periapical lesions is dependent on various factors, one of which is the type and characteristics of endodontic sealer. By increasing the $\mathrm{pH}$ and producing a tight seal, bioceramic sealers are expected to facilitate the healing process.

Purpose: The aim of this pilot study is to present the healing process of lesions of endodontic origin when BioRoot RCS (Septodont, France) was used as an endodontic sealer.

Materials and Methods: Five cases with lesions of endodontic origin were cleaned and shaped using Pro Taper Gold System, EDTA, $\mathrm{NaOCl}$, and obturated with single cone hydraulic condensation technique using BioRoot RCS.

Results: Cases are followed for at least 18 months. XRays show apposition of bone and recovery of the periodontal space. Our results correspond to the literature data found in available scientific sources.

Conclusion: Bioceramics is the first group of endodontic sealers that exhibit both expansion during setting and also an adhesive bond to the dentinal wall. These properties suggest more predictable results.

Keywords: bioceramic sealer, BioRoot, chronic periodontitis,

\section{INTRODUCTION:}

The healing of periapical lesions is dependent on various factors, one of which is the type and characteristics of endodontic sealer [1]. By increasing the $\mathrm{pH}$ and producing a tight seal, bioceramic sealers are expected to facilitate the healing process of chronic inflammations of endodontic origin. They are highly biocompatible and indicated to be used with a simple obturation technique - single cone hydraulic condensation. They possess good adhesion to the dentinal wall and also antimicrobial properties [2]. After direct contact with BioRoot RCS, the viability of periodontal ligament (PDL) cells and their capacity to secrete significant levels of osteogenic and angiogenic factors are preserved [3]. Due to their recent introduction into clinical practice, more studies are needed to prove the clinical outcome.
BioRoot RCS is one of the newest bioceramic sealers on the market. It comes in the form of powder and liquid and is designed to be mixed by simple spatulation, without the need for a special mixing machine. It sets much faster than the previous generations of bioceramics - only 4 hours for complete setting, due to the calcium chloride included in the liquid part. The working time is around 15 minutes, which is more than enough for application in the clinical environment. The consistency of the paste is very smooth, with good flow and good adhesion to the instruments. The radiopacity is achieved by replacing the previously used bismuth oxide with zirconium oxide, which is biocompatible and does not cause discolouration. The Active Biosilicate Technology ensures a medical grade of purity of the calcium silicate content. [4]

There is a limited number of studies concerning the ability to remove these materials from the canal in cases of endodontic retreatments. Radeva concludes that neither hand nor machine driven techniques were able to completely remove the material from the canal walls [5].

\section{PURPOSE:}

The aim of this pilot study is to present the healing process of lesions of endodontic origin when BioRoot RCS (Septodont, France) was used as an endodontic sealer.

\section{MATERIALSAND METHODS:}

Five cases with lesions of endodontic origin were cleaned and shaped using Pro Taper Gold System, EDTA, $\mathrm{NaOCl}$, and obturated with single cone hydraulic condensation technique using BioRoot RCS.

\section{Case 1.}

A 22-years young woman came to the dental office with complaints of occasional pain on chewing on the right side. On visual inspection, we noticed a big composite restoration on her first right mandibular molar. The tooth was tender only to vertical percussion, and no periodontal pockets were found. The sectional X-Ray (fig. 1-1) revealed a massive lesion around the mesial root, involving the apical and furcational area, and another, lateral one, located on the mesial side of the same root. No evidence of a prior root canal treatment was found. After seeing the radiographic 
image we checked again for periodontal pockets, but none were found. The lesion was diagnosed to be of an endodontic origin (Periodontitis chronica granulomatosa diffusa sine fistulae 46) and root canal treatment (RCT) was selected as a treatment method. After placement of a rubber dam, we accessed the pulp chamber. No anaesthesia was applied. After initial flaring of the canals working length (WL) was determined using an apex-locator and K-files \#10 for the mesial canals and \# 15 for the distal. Canals were shaped using Pro Taper Universal (PTU) up to F2 $(\mathrm{d}=25)$. Irrigation was performed with $5,25 \%$ sodium hypochlorite $(\mathrm{NaOCl})$ and 17\% EDTA, and physiological solution was used for a final rinse. The canals were then dried using sterile paper points. Cold single cone technique with matching gutta percha points (hydraulic condensation) was used as an obturation method. BioRoot RCS was mixed according to the manufacturer's instructions and applied in each canal, and the F2 gutta percha (GP) points were slowly introduced to full working length. They were then cut using a heat carrier (System B) at the level of the orifice and compacted using a hand plugger. The tooth was sealed using temporary cement (Coltosol-F, Coltene). An X-Ray was done for control of the obturation (fig. 1-2). On the next visit, the tooth was restored with a direct composite Filtek Z550 (3M).
Fig. 1-1. Pre-Op

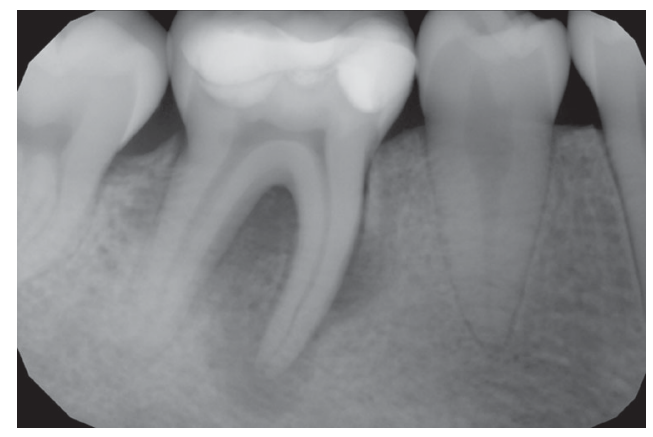

Case 2.

The same young woman had another complaint. She has noticed that a tooth or restoration had chipped and she was getting food entrapped in that area. Upon visual examination, we saw a cavity on the distal aspect of her lower left second molar. Part of the restoration was still present, and the gums in the area were inflamed. Percussion tests were negative, and probing depths were normal. The defect was deep, and we did a sectional X-Ray (fig 2-1). The image showed sub-optimal root canal filling and a localised lesion around the apex of the mesial root. The diagnose was Periodontitis chronica granulomatosa localisata 36, and or-
Fig. 1-2. Post-Op

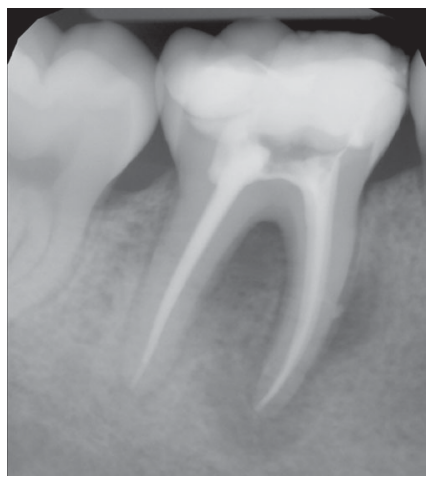

Fig. 1-3. Follow-Up

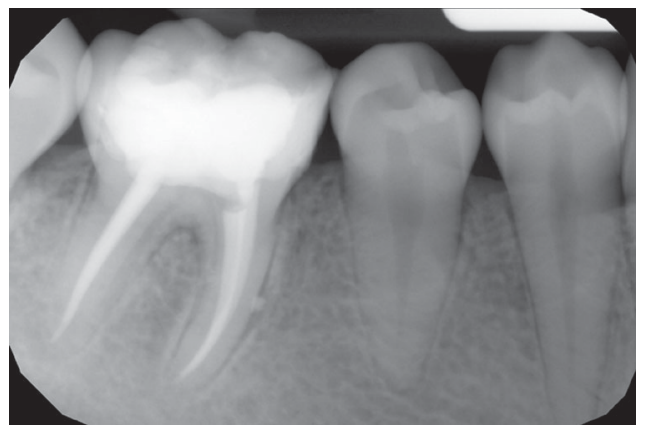

thograde endodontic retreatment was selected as a treatment method. Anaesthesia was applied in the bases of the distal gums papillae, and pre-endodontic build-up was performed. After isolation with a rubber dam, all canals were negotiated using stainless steel (SS) K-files and then shaped using PTU up to F2 for the mesials and F3 for the distal. Irrigation and obturation were performed in the same manner as Case 1. The control X-Ray showed extrusion of material out of the distal canal, but since the GP point F3 was at working length, we decided not to fix the obturation (fig. 2-2). On the next visit, the tooth was restored with a direct composite Filtek Z550 (3M).

Fig. 2-1. Pre-Op

Fig. 2-2. Post-Op

Fig. 2-3. Follow-Up
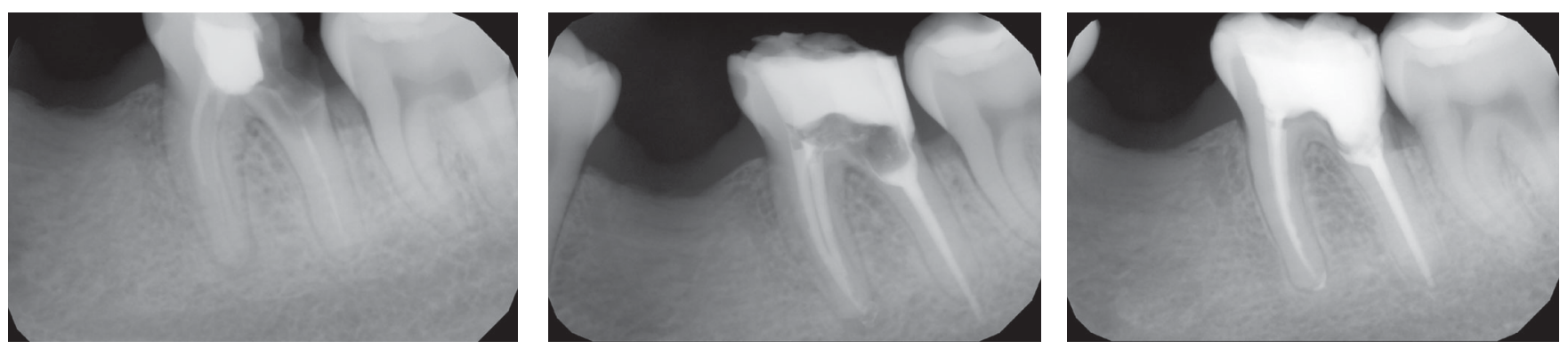

Case 3.

A 45-year male patient came to the dental office with vague sensations somewhere in the lower left. There was a bridge construction, and an X-Ray was done to evaluate the periapical status. The Image showed a non-endodontically treated tooth with a periapical lesion (Periodontitis chronica granulomatosa localisata 34). The patient was happy with the bridge and the decision was made to treat the tooth through the construction. An access cavity was prepared, and WL was determined using an apexlocator. The canal was shaped with PTU up to F2 and irrigated with 5,25\% NaOCl, 17\% EDTA and physiological solution. After drying with paper points, BioRoot was applied in the canal, and GP F2 was slowly placed to WL. An 
X-Ray confirmed the quality of the obturation (fig. 3-1). The access cavity was then filled with a bulk-fill compos- ite material (3M), and the ceramic part was restored using Filtek Z550.
Fig. 3-1. Pre-Op

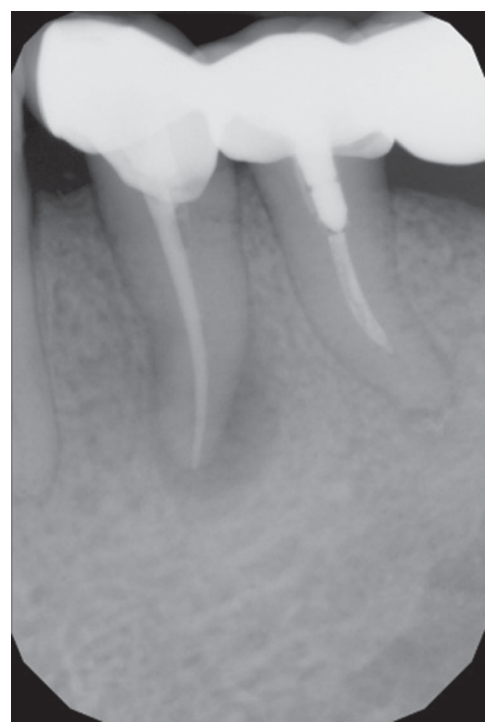

\section{Case 4.}

A 39-years old male patient was referred to our office. A colleague had started an endodontic treatment, but after breaking a small SS hand file at the apex, he decided to refer. We did an X-Ray and saw the little fragment at the very apex of the distal canal of a lower right second molar (fig 4-1). Our diagnose was Periodontitis chronica granulomatosa localisata 47 . Surgery in this region is very
Fig. 3-2. Post-Op
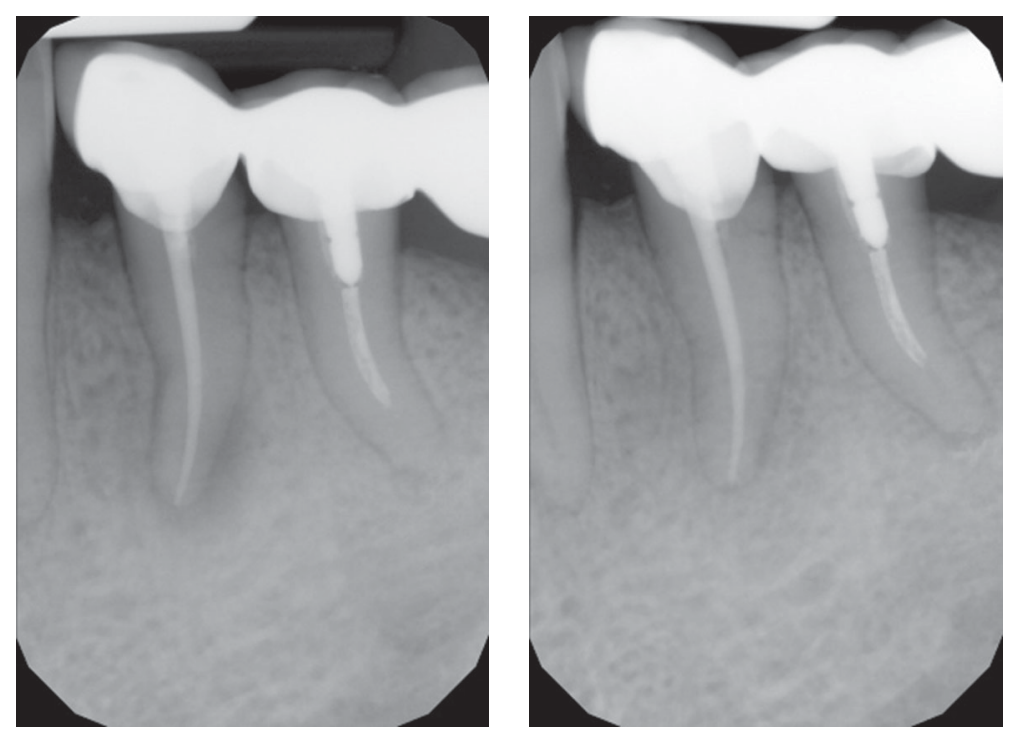

difficult, and the patient rejected that option. After proper isolation with a rubber dam and a lot of struggle, we managed to bypass the fragment. Canals were prepared up to F2 for the mesials and F3 for the distal. Irrigation and obturation was performed in the same manner as Case 1. Control $\mathrm{X}$-Ray was made (fig. 4-2) and restoration was completed with direct composite Filtek Z550.
Fig. 4-1. Pre-Op

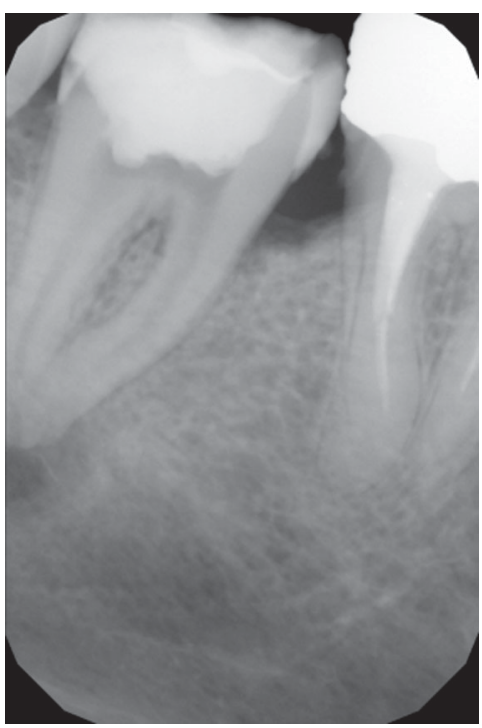

\section{Case 5.}

A 45-years old male patient came to our office with a history of swelling in the gums above his frontal teeth. On examination, we found a scar of fistula in the area of his upper left incisors. His upper left lateral incisor was more tender to vertical percussion than the central or the canine. On palpation, he had minor sensations around the
Fig. 4-3. Follow-Up
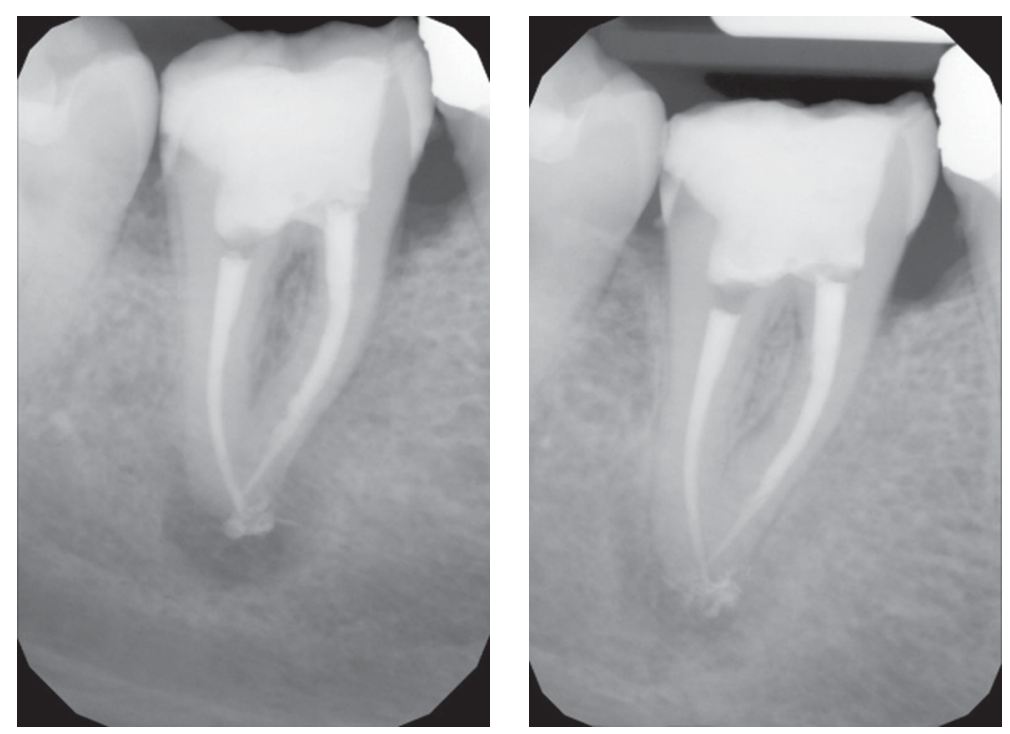

apex of the left lateral incisor. No probing depths were found. On the X-Ray, we found evidence of previous endodontic treatment with sub-optimal root filling and a periapical radiolucency around the apex of his lateral incisor (fig. 5-1). The diagnose we established was Periodontitis chronica granulomatosa diffusa sine fistulae 22. Orthograde endodontic retreatment was carried out in the same man- 
ner as in Case 2. The canal was shaped up to F2 and obturated using matching GP point and BioRoot RCS. After removal of excess and compacting of the GP point, light

Fig. 5-1. Pre-Op

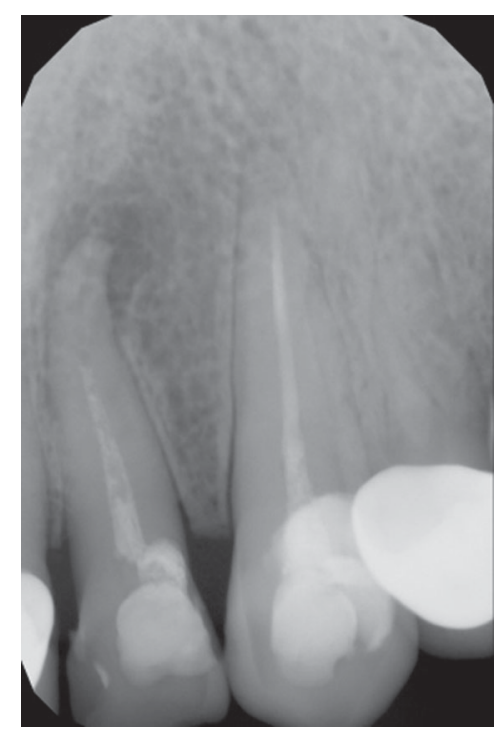

\section{RESULTS:}

Cases are followed for at least 18 months. The teeth are asymptomatic, and X-Rays show apposition of bone and recovery of the periodontal space. Our results correspond to the literature data found in available scientific sources. All the teeth are asymptomatic and completely functional. No pain or tenderness on percussion were detected on the control examinations. Radiographically there is evidence of new bone apposition and disappearing of the pathological radiolucencies (fig. 1-3; 2-3; 3-2; 3-3; 4$3 ; 5-2$ ). No signs of inflammation could be found neither clinically nor radiographically.

\section{DISCUSSION:}

What are the advantages of bioceramics in dental applications? As Koch and Brave suggest in their article "Bioceramic technology - the game changer in endodontics" they are many. [6]

The first advantage is their biocompatibility - these materials are non-toxic to the human cells. [3, 7] They cause little, if any, inflammatory response and are chemically stable within the biological environment, even when an overfill occurs. The latter is not infrequently noticed since chronic periodontitis is often accompanied by resorptive processes of the apical dental tissues, causing an absence of the physiological constriction. (Cases 2,4 and 5) $[1,8,9]$. Gusiyska overviews the nature of the periapical resorptive processes and concludes that if an appropriate sealer is chosen, the healing occurs with the formation of cement-like tissue [1]. Seltzer and Bender stress the importance of the available $\mathrm{Ca}^{2+}$ for the activation of adenosine triphosphatase, which induces the remineralization potential of dental tissues [2].

The second great advantage of this group of materi- cured composite was used for restoration. The patient refused a second X-Ray on the same day, and we managed to get an X-Ray when he came back two years after the retreatment.

Fig. 5-2. Follow-Up

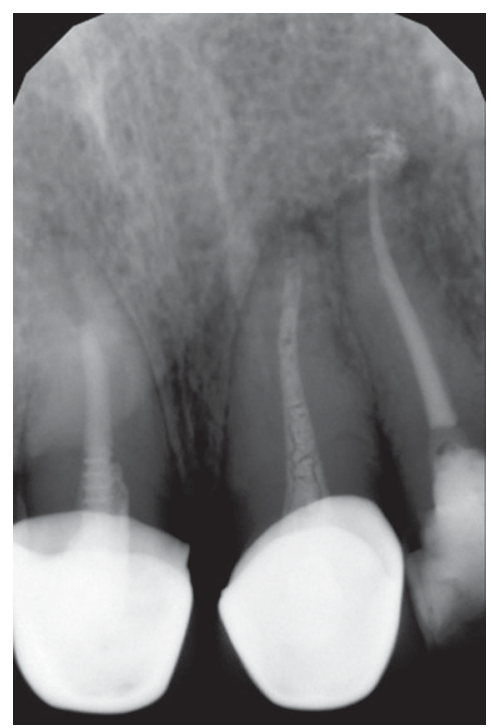

als is their tight bond to the dentinal wall. This is a not fully studied mechanism of both micro-mechanical and chemical adhesion to dentin, which provides a very trustful and long lasting bond. Their hydrophilic nature reduces the contact angle of the sealer and facilitates its penetration into the fine areas of the root canal system. They not only do not shrink but even exhibit slight expansion during setting, which provides exceptional mechanical interlocking. The strong alkalinity of the sealer denatures the collagen fibres, allowing the mineral content of the material to enter diffusely into the intertubular dentine, producing the so-called "mineral infiltration zone" (MIZ). Along with the MIZ, there is also a partial reaction of phosphates with calcium silicate hydrogel and calcium hydroxide, which results in a true chemical ion-to-ion covalent bond. [4, 10, 11, 12, 13]

During setting calcium hydroxide is released as a byproduct of the reaction, providing these materials with antibacterial properties. According to the agar diffusion test, and direct contact testing the antimicrobial properties are high only during setting. After the material is completely set, the $\mathrm{pH}$ is decreased to neutral, and these properties cease to exist. [4, 13, 14, 15]

The current golden standard for endodontic obturation is the continuous wave of condensation - an improved variation of the warm vertical compaction technique, developed by $\mathrm{H}$. Schilder. $[4,6,16]$ This technique is very complex and time-consuming. On the contrary, the single cone hydraulic condensation technique is simple and fast. It does not require the usage of high temperatures, metal pluggers, or extensive loss of coronal tissues. The obturation is done by the sealer itself, and the gutta percha point is used as a plunger to push the sealer into the fine parts of the canal and in cases of retreatment to act as a path to the 
apical foramen. $[4,16]$

All of the above mentioned qualities of the bioceramic endodontic sealers rises them as a promising alternative to the present golden standard in endodontic obturation.

As for the disadvantages very few can be found. One is the unpredictability to be used in conjunction with warm methods for root canal obturation. Boyadzhieva studies the effects of different temperatures on the physical qualities of bioceramic endodontic sealers and proves that they are adversely changed. The heat evaporates the moisture from the setting paste, which desiccates the material, making it brittle and non-homogeneous. This worsens the bond between the material and the dentinal wall and the final stability of the set material. [17].

Another one is the difficulty of their removal in cases of orthograde retreatment. Only two studies were found in the available literature concerning the removal of BioRoot RCS. In both of them, the bioceramic sealer was sufficiently removed from the canal walls during retreatment procedures. There is no specific solvent for these types of materials developed yet, so the other types of sealers were more efficiently removed with the help of solvents. [18, 19]

\section{CONCLUSION:}

Bioceramics is the first group of endodontic sealers that exhibit both expansion during setting and also an adhesive bond to the dentinal wall. These properties suggest more predictable results.

Although the results with this new type of materials are very promising, it is the lack of sufficient clinical studies that stops many experienced clinicians to switch to this new concept of obturation. Clinical follow-ups are the strongest prove of the outcome of the treatment.
Abbreviations:
PDL - periodontal ligament
RCT - root canal treatment
WL - working length
PTU - Pro Taper Universal
GP - gutta percha
SS - stainless steel
MIZ - mineral infiltration zone

\section{REFERENCES:}

1. Gusiyska A. Periapical resorptive processes in chronic apical periodontitis: an overview and discussion of the literature. J of IMAB. 2014 Oct-Dec;20(5):601-5. [Crossref]

2. Ehrmann EH. Endodontology Biologic Considerations in Endodontic Procedures: S. Seltzer. Australian Endodontic Newsletter. 1990 Apr; 15(3):14-15.

3. Camps J, Jeanneau C, El Ayachi I, Laurent P, About I. Bioactivity of a calcium silicate-based endodontic cement (BioRootTM RCS): interactions with human periodontal ligament cells in vitro. JOE. 2015 Sep;41(9): 1469-73

4. Simon S, Flouriot AC. Bi oRoot $^{\mathrm{TM}} \mathrm{RCS}$, a new biomaterial for root canal filling. 2016 [ Internet]

5. Marinova-Takorova M, Radeva E, Kisyova I, Naseva E. Retreatment of teeth filled with single cone technique and MTA based sealer - comparison of the effectiveness of different retreatment techniques (in vitro study). J of IMAB. 2019 JanMar;25(1):2379-83. [Crossref]

6. Koch K, Brave D. Bioceramic Technology-the game changer in endodontics. Endodontic Practice 2009 Jan;2:17-21.

7. Dimitrova-Nakov S, Uzunoglu
E, Ardila-Osorio H, Baudry A, Richard $\mathrm{G}$, Kellermann $\mathrm{O}$, et al. In vitro bioactivity of Bioroot ${ }^{\mathrm{TM}} \mathrm{RCS}$, via A4 mouse pulpal stem cells. Dent Mater. 2015 Nov;31(11):1290-7

8. Chybowski E A, Glickman G N, Patel Y, Fleury A, Solomon E, He J. Clinical Outcome of Non-Surgical Root Canal Treatment Using a Singlecone Technique with Endosequence Bioceramic Sealer: A Retrospective Analysis. JOE 2018 Jun;44(6):941945.

9. Zaki D Y, Zaazou M H, Khallaf M E, Hamdy T M. In Vivo Comparative Evaluation of Periapical Healing in Response to a Calcium Silicate and Calcium Hydroxide Based Endodontic Sealers. Open Access Maced J Med Sci. 2018 Aug 20;6(8):1475-79.

10. Atmeh A R, Chong E Z, Richard G, Festy F, Watson T F. Dentin-cement Interfacial Interaction: calcium silicates and Polyalkenoates. J Dent Res. 2012 May;91(5):454-9.

11.Xuereb M, Vella P, Damidot D, Sammut C V, Camilleri J. In Situ Assessment of the Setting of Tricalcium Silicate-based Sealers Using a Dentin Pressure Model. JOE 2015 Jan; 41(1):111-24.

12.Parirokh M, Torabinejad M. Mineral Trioxide Aggregate: A Com- prehensive Literature Review-Part I: Chemical, Physical, and Antibacterial Properties JOE 2010 Jan;36(1):16-27.

13. Al-Haddad A, Che Ab Aziz ZA. Bioceramic-Based Root Canal Sealers: A Review. Int J Biomater. 2016; 2016: 9753210. [PubMed] [Crossref]

14. Wang Z, Shen Y, Haapasalo M. Dental materials with antibiofilm properties. Dental Materials 2014; 30:e1-e16.

15.Zhang H, Shen Y, Ruse D N, Haapasalo M. Antibacterial Activity of Endodontic Sealers by Modified Direct Contact Test Against Enterococcus faecalis. JOE $2009 \mathrm{Jul} ; 35(7)$ : 1051-55.

16. Cohen RG. Single point gutta percha obturation using a tricalcium silicate endodontic sealer. [Internet]

17. Boyadzhieva E. [Application of bioceramic sealer in obturation of the root canal space with thermoplastified guttapercha.] [Dissertation]. Medical University - Plovdiv. 2018. [in Bulgarian]

18. Kakoura F, Pantelidou O. Retreatability of root canals filled with Gutta percha and a novel bioceramic sealer: A scanning electron microscopy study. J Conserv Dent. 2018 Nov-Dec;21(6):632-636.

19. Donnermeyer D, Bunne C, 
Schafer E, Dammaschke T. Retreatability of Three Calcium SilicateContaining Sealers and One Epoxy Resin-Based Root Canal Sealer With Four Different Root Canal Instruments. Clin Oral Investig. 2018 Mar; 22(2):811-817. [PubMed] [Crossref]

Please cite this article as: Zhekov K, Stefanova V. Healing of Chronic Apical Periodontitis Using Novel Bioceramic Sealer BioRoot RCS. J of IMAB. 2020 Apr-Jun;26(2):3081-3086. DOI: https://doi.org/10.5272/jimab.2020262.3081

Received: 27/06/2019; Published online: 21/04/2020

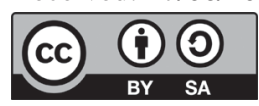

Address for correspondence:

Dr. Kostadin Zhekov,

Department of Operative Dentistry and Endodontics, Faculty of Dental Medicine, Medical University - Plovdiv

E-mail: ko.zhekov@gmail.com 